\title{
XMM-Newton Publication Statistics
}

\author{
J.-U. Ness ${ }^{1, \star}$, A.N. Parmar ${ }^{2}$, L.A. Valencic ${ }^{3,4}$, R. Smith ${ }^{5}$, N. Loiseau ${ }^{1}$, A. Salama ${ }^{1 \star \star}$, M. Ehle ${ }^{1}$, and N. \\ Schartel ${ }^{6}$ \\ 1 XMM-Newton Science Operations Centre, Science Operations Department of ESA, ESAC, 28691 Villanueva de la \\ Cañada (Madrid), Spain; e-mail: juness@ sciops.esa.int \\ 2 Science Support Office, Directorate of Science and Robotic Exploration, ESA/ESTEC, Postbus 299, NL-2200 AG \\ Noordwijk, The Netherlands \\ 3 Johns Hopkins University, Baltimore, MD 21218 \\ ${ }^{4}$ NASA Goddard Space Flight Center, Greenbelt, MD 20771 \\ 5 Harvard Smithsonian Center for Astrophysics, 60 Garden Street, Cambridge, MA 02138 USA \\ 6 Astrophysics and Fundamental Physics Missions Division, Research and Scientific Support Department of ESA, ESAC, \\ Villanueva de la Cañada (Madrid), Spain
}

Received 31 July 2013

Published online later

Key words GENERAL - publications, bibliography

We assessed the scientific productivity of XMM-Newton by examining publications and data usage statistics. We analyse 3272 refereed papers, published until the end of 2012, that directly use XMM-Newton data. The SAO/NASA Astrophysics Data System (ADS) was used to provide additional information on each paper including the number of citations. For each paper, the XMM-Newton observation identifiers and instruments used to provide the scientific results were determined. The identifiers were used to access the XMM-Newton Science Archive (XSA) to provide detailed information on the observations themselves and on the original proposals. The information obtained from these sources was then combined to allow the scientific productivity of the mission to be assessed. Since around three years after the launch of XMMNewton there have been around 300 refereed papers per year that directly use XMM-Newton data. After more than 13 years in operation, this rate shows no evidence that it is decreasing. Since 2002, around 100 scientists per year became lead authors for the first time on a refereed paper which directly uses XMM-Newton data. Each refereed XMM-Newton paper receives on average around four citations per year in the first few years with a long-term citation rate of three citations per year, more than five years after publication. About half of the articles citing XMM-Newton articles are not primarily X-ray observational papers. The distribution of elapsed time between observations taken under the Guest Observer programme and first article peaks at 2 years with a possible second peak at 3.25 years. Observations taken under the Target of Opportunity programme are published significantly faster, after one year on average. The fraction of science time taken until the end of 2009 that has been used in at least one article is $\sim 90 \%$. Most observations were used more than once, yielding on average a factor of two in usage on available observing time per year. About $20 \%$ of all slew observations have been used in publications. The scientific productivity of XMM-Newton measured by the publication rate, number of new authors and citation rate, remains extremely high with no evidence that it is decreasing after more than 13 years of operations.

(c) 2006 WILEY-VCH Verlag GmbH \& Co. KGaA, Weinheim

\section{Introduction}

XMM-Newton was launched on 10 December 1999 into a 48-hour highly elliptical orbit. The mission provides sensitive X-ray imaging and spectroscopic observations of a wide variety of cosmic sources from nearby solar system objects to the most distant black holes (Jansen et al. 2001). The payload consists of the European Photon Imaging Camera (EPIC), the Reflection Grating Spectrometer (RGS; den Herder et al. 2001) and the Optical Monitor (OM; Mason et al. 2001). The EPIC consists of 3 imaging spectrometers each located at the focus of an X-ray optic consisting of 58 nested Wolter I geometry mirrors. Two of

\footnotetext{
^ Corresponding author: e-mail: juness@ sciops.esa.int

$\star \star$ A.S. sadly deceased before the project was completed
}

the EPIC cameras are based on MOS-CCD technology and share the mirrors with RGS grating arrays (Turner et al. 2001) while the detector based on pn-CCD technology is located behind a fully open telescope (Struider et al. 2001). The overall effective aperture is $4500 \mathrm{~cm}^{2}$ at $1 \mathrm{keV}$ and the spatial resolution is 15 arc seconds (half-energy width) with a field of view of $\sim 30$ arc minutes diameter. The RGS provides $0.35-2.4 \mathrm{keV}$ spectra with an $\mathrm{E} / \Delta \mathrm{E}$ of $300-700\left(1^{\text {st }}\right.$ order). The effective area for the two grating arrays varies in the range of $40-200 \mathrm{~cm}^{2}$ over the energy range. The OM provides optical and UV monitoring of fluxes through various filters as well as spectroscopy with two grisms. Normally, all three instruments are operated simultaneously.

XMM-Newton observing time is allocated in different ways: 
- The majority of the observing time is available to the world-wide scientific community in the Guest Observer (GO) programme. Targets are selected competitively through peer review by a Time Allocation Committee which evaluates proposals. The calls for proposals are normally issued annually and are typically six times oversubscribed in terms of available observing time. Investigator teams of successful proposals are granted a proprietary period of one year before the data are made publicly available.

- During the first two years of the mission, the scientific groups that were involved in the instrument and scientific ground segment development were awarded Guaranteed Time observations.

- XMM-Newton undertakes a small number of calibration observations which are made immediately accessible to the public.

- Target of Opportunity (ToO) observations allow XMMNewton to respond to unique events that sometimes require quick reaction. Members of the scientific community can alert the Project Scientist who may approve observations after evaluation.

The scientific products from each observation, produced by a pipeline processing, are stored in the XMM-Newton Science Archive (XSA). During any proprietary period, data in the XSA are only made available to the Principal Investigator (PI) of the observation, otherwise all the products stored in the XSA are available for public download via the internet. The XSA has more than 3000 registered users of which around 1000 download data each year. General information such as observing date and time, instrument use, name of PIs etc. can be accessed by the general public for all observations in the XSA. The key to access a data set belonging to a specific observation is the unique 10-digit observation identifier (ObsID).

Here we investigate the scientific productivity of the XMM-Newton observatory. We have linked the papers to the XMM-Newton observations that were used to provide the data that were analysed. XMM-Newton has a large number of publications (currently over 3500 refereed papers) which have resulted from data obtained over more than 13 years of observations and published by more than 1200 first authors. In the following, we describe the criteria by which publications are included in the XMM-Newton publication list, the process by which a publication database is populated with the necessary information such as the bibliographic codes, instruments and observations that were used in each paper. We then use this publication database and the links to the data to investigate the scientific productivity of XMM-Newton.
Table 1 Publication classification scheme used in this paper (following Apai et al. 2010 and Rots et al. 2012)

\begin{tabular}{ll}
\hline \hline Category & Description \\
\hline 1 & $\begin{array}{l}\text { The publication makes direct use of XMM-Newton } \\
\text { data }\end{array}$ \\
2 & The publication refers to published results \\
3 & The publication predicts XMM-Newton results \\
4 & The publication describes XMM-Newton \\
& instrumentation, software, or operations \\
5 & Other XMM-Newton related articles \\
\hline
\end{tabular}

\section{Generation of the XMM-Newton publication database}

\subsection{XMM-Newton publication list}

The first step is to define the papers that are to be considered as XMM-Newton papers for this study. Apai et al. (2010) and Rots et al. (2012) have defined five categories of mission-related publications which are listed in Table 1 .

Since the start of the XMM-Newton mission, the XMM-Newton Guest Observer Facility, at the Goddard Space Flight Center (GSFC), has kept a detailed record of the bibcodes of all refereed XMM-Newton publication 1 . Published articles are considered as XMM-Newton papers if they are published in refereed journals, mention "XMM-Newton" or "X-ray" in the abstract, and include XMM-Newton data or results. This can be done either through directly reducing and analysing XMM-Newton data, discussing in detail previous XMM-Newton results, using data obtained from XMM-Newton in plots or text, or using XMM-Newton survey results.

We include in this study only category I papers from Table 11, those that make direct use of XMM-Newton data including X-ray fluxes or hardness ratios from the 2XMM catalogue (Watson et al. 2009) and the slew survey (Saxton et al. 2008). We also add papers from the special issue of Astronomy \& Astrophysics (vol. 365) in 2001 which included the descriptions of the mission, instruments and ground segment (category 4 in Table 1 ).

The GSFC archive of XMM-Newton papers is regularly updated with newly published papers. Since the GSFC list includes all the publication categories listed in Table 1, the XMM-Newton Project Scientist at ESA manually checks each listed paper to select those that fulfil the criteria discussed above. The list of papers derived in this way is then used to provide information to ESA management and elsewhere. As of autumn 2013, there are more than 3500 refer-

\footnotetext{
${ }^{1}$ http://heasarc.gsfc.nasa.gov/docs/xmm/xmmbib.html
} 
eed papers that fulfil the criteria listed above out of which we analyse 3272 that were published until the end of 2012.

\subsection{Observation identifier (ObsID) determination}

The next step is to derive the XMM-Newton observation identifiers (ObsIDs) for the observations that were analysed to provide the scientific results in each paper. These, and the instruments used, were extracted manually from the papers with the help of the other scientists given in acknowledgements. Whilst the XMM-Newton publication guideline 2 request that the $\operatorname{Obs} \operatorname{ID}(\mathrm{s})$ and instruments be included in the article, only about $40 \%$ of papers actually contain this information. Observations that are not used directly for scientific purposes but, e.g., for determination of instrumental background are not included in the database. For the majority of papers which did not provide ObsIDs this was still a relatively straight forward process, utilising information such as the target name, coordinates, observation date, or instrumental setup if provided. Unique catalogue names from the 2XMM catalog Watson et al. 2009) and the slew survey (Saxton et al. 2008), if given by the authors, allow extraction of the corresponding ObsIDs from the catalog.

Care was taken with papers that used large numbers of observations such as sky surveys, for which observations have been selected by the authors from criteria that are not uniquely reproducible. With the co-operation of the corresponding authors, most of these papers (38) could be resolved, although some uncertainty of the order 5\% remains. We flagged 29 difficult papers for which the ObsIDs included in the database are particularly uncertain. To minimise human errors, each article was screened independently by scientists in the XMM-Newton Guest Observer Facility at the Goddard Space Flight Center (GSFC), USA and in the XMM-Newton Science Operations Centre (SOC) in Spain. The results from both groups were then compared and discrepancies resolved.

\subsection{Publication database}

Information for each XMM-Newton article is stored in a database whose contents are given in Table 2 The bibcodes allow the ADS to be accessed and the information listed under ADS in Table 2 to be obtained. Similarly the ObsIDs allow the XSA to be accessed and the information listed under XSA to be extracted. In addition, the submission dates were extracted directly from the articles via automatic keyword search $(\sim 95 \%)$ and manual checks $(\sim 2 \%)$. The maximum delay between submission and publication dates listed in the ADS is 4.7 years whilst on average, it is six months. The ADS also allows cross links between publications that make reference to each other which were used to create a citation database of refereed articles citing

${ }^{2} \mathrm{http} / / / \mathrm{xmm}$. esac.esa.int/external/xmm_science/pub_guide.shtml
Table 2 Contents of each entry in the XMM-Newton publication database

\begin{tabular}{|c|c|}
\hline Source & Item \\
\hline NASA GSFC & Publication bibcode \\
\hline $\begin{array}{l}\text { ESA SOC } \\
\& \text { GSFC }\end{array}$ & $\begin{array}{l}\text { ObsID }(\mathrm{s}) \text { of data analysed in paper } \\
\text { Instruments used to provide analysed data }\end{array}$ \\
\hline $\begin{array}{l}\text { Publication d } \\
3272^{a} \text { Paper } \\
3219^{b} \text { Paper } \\
3187^{b} \text { Paper } \\
79348 \text { ObsIl } \\
72634^{b} \text { certs } \\
7131 \text { unique } \\
7096^{b} \text { certai }\end{array}$ & $\begin{array}{l}\text { abase used in this work } \\
\text { analysed in this work } \\
\text { with ObsIDs ( } 98.4 \% \text { of previous) } \\
\text { with }<290 \text { ObsIDs ( } 99.0 \% \text { of previous) } \\
\text { in total } \\
\text { ObsIDs ( } 91.5 \% \text { of previous) } \\
\text { bsIDs } \\
\text { unique ObsIDs ( } 99.5 \% \text { of previous) }\end{array}$ \\
\hline ADS & $\begin{array}{r}\text { Publication Date } \\
\text { Journal name } \\
\text { Authors name(s) } \\
\text { Paper title and abstract } \\
\text { bibcode(s) of citing paper(s) }\end{array}$ \\
\hline \multicolumn{2}{|c|}{$\begin{array}{l}\text { Citation database of refereed citing articles } \\
\text { published until } 10 \text { October } 2013 \\
74564^{a} \text { Refereed citing papers } \\
20295^{a} \text { unique citing papers }\end{array}$} \\
\hline 8577 usable & $\begin{array}{r}\text { Observation date } \\
\text { Exposure time } \\
\text { Name(s) of proposers } \\
\text { Proposed science category } \\
\text { servations (see Sect. 3] }\end{array}$ \\
\hline $\begin{array}{l}\text { Used in Sect. } \\
\text { Used in Sect. }\end{array}$ & \\
\hline
\end{tabular}

XMM-Newton articles. We analyse only papers published until the end of 2012 whilst we include citations that were included in the ADS until 10 October 2013. The citation database was populated by extracting for each article in the publication database a list of bibcodes from the ADS that belong to refereed articles referring to it.

The contents of the ADS originate from metadata provided by the journals which in turn receive the information from the authors when submitting the papers. References may be incomplete due to the inability to match them with $100 \%$ accuracy (e.g. in press, private communications, author errors, etc.). The robustness of citations in the ADS was compared to the Science Citation Index (SCI) by H. A. Abt (published in Heck 2006/3) and demonstrates the power of ADS with $15 \%$ more citations than SCI and missing less than $1 \%$ of the citations. Some caveats when analysing citations as criterion to assess the impact of an article are described, e.g., in Rots et al. (2012), and only rough measures are possible to deal with contaminating effects such as self citations. However, a systematic study

\footnotetext{
3 http://www.garfield.library.upenn.edu/papers/... ...helmutabtorgstratastronv6y2004.html
} 
presented by Trimble (1986) analysing articles that were published during January 1983 revealed that only about $15 \%$ of all citations were actually self citations and that the rate varies very little (8\%) among 33 world wide journals. In light of the complexity of any corrective measure and reproducibility of results, we use the citation statistics as they are extracted from the ADS.

\section{Analysis}

The bibcodes and ObsIDs allow access to the ADS and the XSA to provide details about the papers and the observations, respectively. In Sect.4.1, we present results that rely only on information extracted from the ADS which allows items such as publication rate, journal statistics, author statistics, and citation statistics to be investigated. Although the ADS operates a sophisticated system that streamlines author names, the identification of individual authors is not straightforward. We identified individuals by their full last name and first initial. This will count different authors with common last names such as John Smith and Jim Smith as a single author which is a conservative approach for our purposes. On the other hand, we avoid the larger number of same authors with different entries of first name such as J. Smith, J.H. Smith, John H. Smith, J. Hamilton Smith, etc. to be counted multiple times. Double counts can also arise from different spellings of last names, in particular if they contain special characters such as Spanish or French accents or German umlauts. We perform systematic replacements of all special characters in the ADS lists of authors by utf- 8 compliant characters to minimise the double counting of different authors.

To assess the usage of XMM-Newton data presented in Sect. 4.2, we determined the elapsed time between the observation and first publication of the data, the fraction of the observing time used in publications, multiple use of the same XMM-Newton observation and usage of data from different science programmes and science categories. The data for these studies originates from the combined information obtained by linking the bibcodes and ObsIDs. This linking allows publication dates and citation statistics obtained from the ADS to be cross-correlated with observing dates, exposure times, science programmes and categories etc. obtained from the XSA.

The reference sample of useable observations, suitable for scientific analysis, comprises 8577 pointed observations, obtained until the end of 2012. The definition of "useable" is that the automatic generation of the Observation Data File (ODF) was successful and the products from the Pipeline Processing System (PPS) are availale from the current version of the XSA. Observations with only $\mathrm{OM}$ exposures are excluded from the reference sample. We added 94 observations for which no PPS products had been released, but where at least one EPIC camera was operated in a science mode with more than 1000 seconds exposure time. We did not screen the quality reports, nor did we filter on good time intervals. The reference sample is thus a rough estimate of observations that are available to the scientific community and is used to investigate the degree to which the science products were used.

As a measure of the impact of an observation in the reference sample, we determined the number of papers using it, the date when the first paper was published, and the number of refereed papers citing all articles combined using the particular observation.

\section{Results}

The publication database up to the end of 2012 contains in total 3272 articles, out of which we were able to identify ObsIDs for 3219. 29 articles with uncertain lists of ObsIDs were discarded. The citation database contains all refereed articles that were published until 10 October 2013. In Table 2, we give an account of the contents of the publication and citation databases with total number of entries. Those samples are used in subsections 4.1 and 4.2 are marked by superscripts $a$ and $b$, respectively.

\subsection{Publication statistics}

\subsubsection{Publication rates}

Based on the publication dates extracted from the ADS, we determined the number of published papers per calendar year and show the evolution in Fig. 1] Following launch in December 1999, the first refereed papers appeared in 2001. By 2004-2005, a stable publication rate of around 300 papers per year had been reached. It is remarkable that the publication rate shows no evidence for any reduction, even more than 13 years after launch.

\subsubsection{Publication journals}

The most popular journal in which to publish XMMNewton papers is Astronomy \& Astrophysics (A\&A) followed by the Astrophysical Journal (ApJ), and Monthly Notices of the Royal Astronomical Society (MNRAS); 87\% of all XMM-Newton papers have been published in one of these three journals. Extending this to the nine journals listed in Table 3 allows almost $96 \%$ of all XMM-Newton refereed papers to be included. The last two columns in Table 3 give the number and percentage of papers in each journal that provide references to the XMM-Newton papers. Here, papers citing more than one XMM-Newton papers are counted only once. Whilst the majority of XMMNewton papers are published in A\&A, papers appearing in ApJ make the highest number of references to them. 


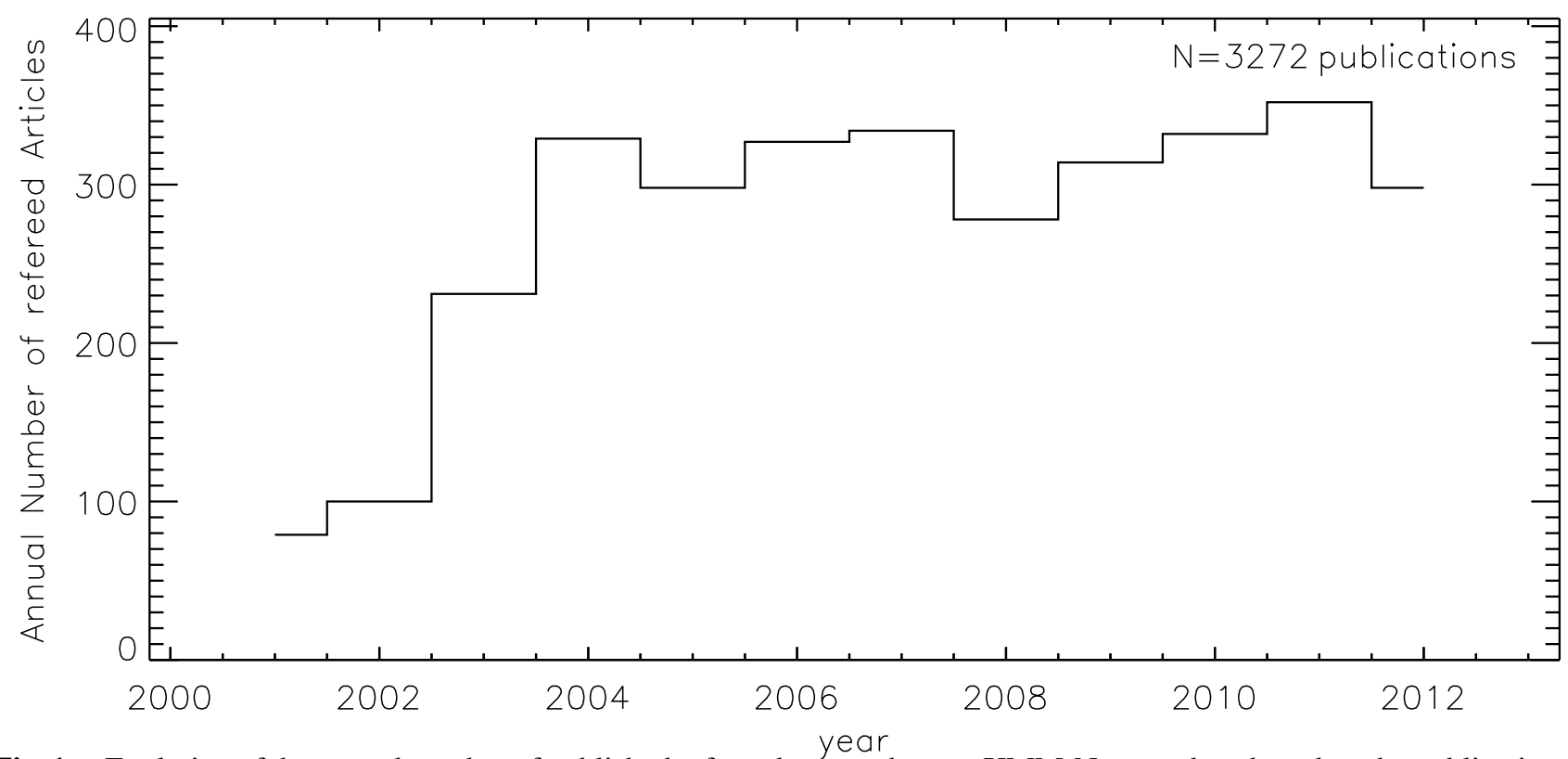

Fig. 1 Evolution of the annual number of published refereed papers that use XMM-Newton data, based on the publication dates extracted from ADS. Since 2003, around 300 new XMM-Newton papers are published every year.

Table 3 Nearly $96 \%$ of all XMM-Newton refereed papers are published in the nine journals listed. The journal abbreviations used in the bibcodes are given in brackets.

\begin{tabular}{|c|c|c|c|c|}
\hline Journal & $\begin{array}{l}\text { Number of } \\
\text { publications }\end{array}$ & $\%$ & $\begin{array}{l}\text { Number of } \\
\text { citations }^{a}\end{array}$ & $\%$ \\
\hline Astronomy \& Astrophysics (A\&A) & 1170 & 35.8 & 4002 & 19.7 \\
\hline Astrophysical Journal (ApJ) & 1015 & 31.0 & 7478 & 36.8 \\
\hline $\begin{array}{l}\text { Monthly Notices of the Royal } \\
\text { Astronomical Society (MNRAS) }\end{array}$ & 659 & 20.1 & 4371 & 21.5 \\
\hline Astronomical Journal (AJ) & 66 & 2.02 & 616 & 3.04 \\
\hline $\begin{array}{l}\text { Publications of the Astronomical } \\
\text { Society of Japan (PASJ) }\end{array}$ & 48 & 1.47 & 400 & 1.97 \\
\hline Astronomische Nachrichten (AN) & 77 & 2.35 & 241 & 1.19 \\
\hline Advances in Space Research (AdSpR) & 72 & 2.20 & 180 & 0.89 \\
\hline Nature + Science & 24 & 0.73 & 229 & 1.13 \\
\hline Total: & 3131 & 95.7 & 17517 & 86.3 \\
\hline
\end{tabular}

${ }^{a}$ as of 10 October 2013

\subsubsection{Authors}

An analysis of first- and co-authors publication rates gives insights into the XMM-Newton user community. For each unique name we determined the dates when he or she first appeared as a first- or co-author of a refereed XMM-Newton paper. The results are shown in Fig. 2 where the number of first and co-authors publishing an XMM-Newton data paper for the first time is shown. The grey histogram indicates all authors, whilst the black histogram counts only first authors. It can be seen that the number of new first authors has remained approximately constant at around 100 scientists each year. The number of new authors may be showing a gradual increase with time. The fact that there are around 100 new first authors each year demonstrates that the XMM-Newton user community continues to grow, demonstrating the continued relevance of the mission to the wider scientific community.

\subsubsection{Citations}

In Table 4 we list the XMM-Newton papers with the highest citation rates, giving bibcodes, first author, instruments used, number of citations, annual citation rate and titles. Only papers with an exposure to the public of more than two years were considered (thus were published until the end of 2010) in order to eliminate short-term effects. The highest citation rates were received by the instrument and 


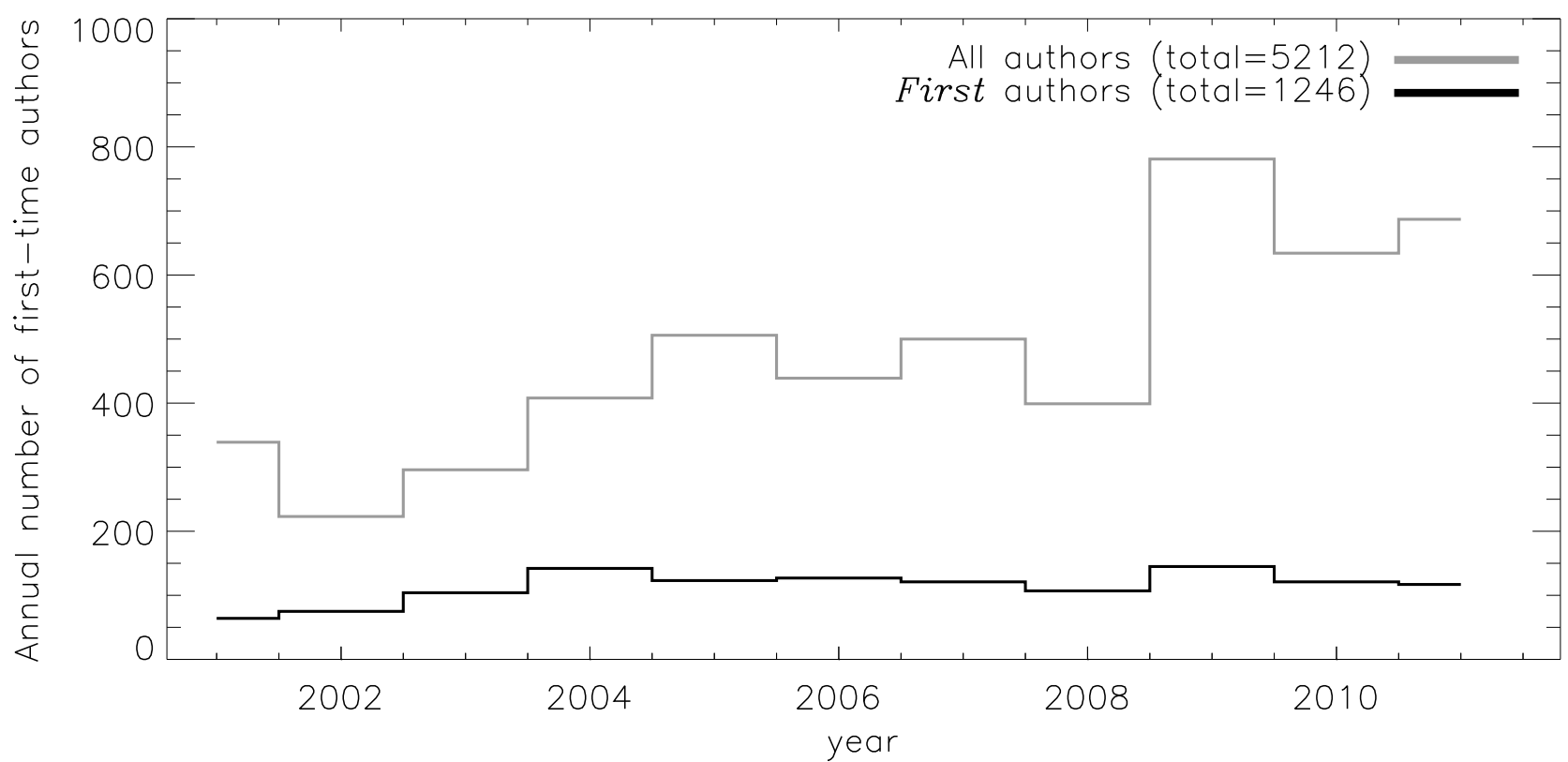

Fig. 2 Evolution with time of new authors (grey) and new first authors (black) publishing a refereed paper which directly uses XMM-Newton data.

2XMM catalog papers.

In Fig. 3, we show the number of citations per year per number of papers published in each corresponding year (=number of citable items). To assess the longer-term impact of the mission we have also determined the fraction of citations that were given more than 2, 3, 4, and 5 years after publication and mark these with different shades of red in Fig. 3 (see legend). The reference time period used to determine the rate is the time between publication and 10 October 2013. For the long-term citation rates, the reference period is accordingly shorter. For example, for a paper published in January 2003, the total reference period is 10 years, whilst the reference period for citations given later than 3 years after publication is only 7 years. In general, papers published shortly after the launch of the mission have received the highest number of citations (note that citations to instrument articles Strüder et al. (2001); Turner et al. (2001); den Herder et al. (2001); Mason et al. (2001); Talavera (2009); Jansen et al. (2001) are excluded here). After 2002, the number of citations has remained at an average rate of 3-4 citations per year per paper, e.g., a paper published in 2003 has on average been cited 38 times. The long-term citation rates for all four reference periods remain remarkably high compared to the shorter-term citation rate. We emphasise that the corresponding citation rates include citing papers from the most recent past which shows the continuing relevance of XMM-Newton results.

In order to estimate the impact of XMM-Newton beyond the X-ray community, we determined the fraction of papers that cite XMM-Newton papers that are not included in our publication database. The evolution of this fraction is shown by the black histogram in Fig. 4, starting with the

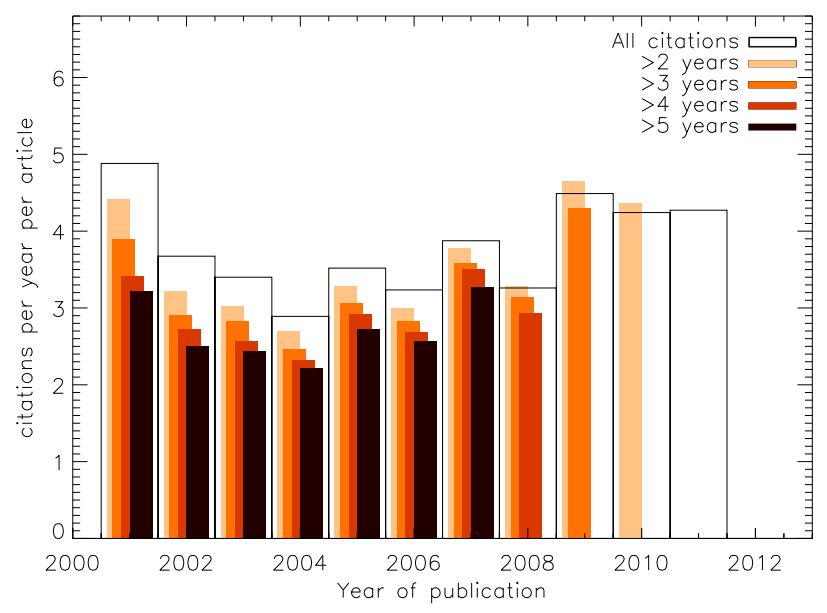

Fig. 3 Combined annual citation rates received by all papers within each year, normalised by the corresponding number of papers in the same time interval. The shaded bars indicate the citation rates by papers that appeared more than $2,3,4$, and 5 years after the cited papers were published to illustrate the long-term impact.

year 2003, when the publication rate has reached a stable level (see Fig. 1). In addition, we determined the (lower) fraction of these articles that do not contain the names of other current major X-ray missions Chandra, Suzaku, or Swift in their titles or abstracts and show the respective fractions with the grey histogram. As more XMM-Newton papers were published, the fraction of non-XMM-Newton papers citing XMM-Newton papers has increased. Also, the fraction of citing papers not primarily analysing X-ray data has increased, having reached over $50 \%$ in 2012 . This shows that the high citation rate is not generated by the $\mathrm{X}$-ray community itself, but that XMM-Newton results are 
Table 4 XMM-Newton Papers with highest citation rates until 10 October 2013

\begin{tabular}{|c|c|c|c|c|}
\hline bibcode & Authors & Instruments & \#citations & citations per year \\
\hline \multirow[t]{2}{*}{ 2001A\&A...365L..18S } & L. Strüder et al. & EPN & 1089 & 84.68 \\
\hline & \multicolumn{4}{|c|}{ The European Photon Imaging Camera on XMM-Newton } \\
\hline \multirow[t]{2}{*}{ 2001A\&A...365L..27T } & M. J. L. Turner et al. & EMOS & 1013 & 78.77 \\
\hline & \multicolumn{4}{|c|}{ The European Photon Imaging Camera on XMM-Newton } \\
\hline \multirow[t]{2}{*}{ 2001A\&A...365L ...1J } & F. Jansen et al. & XMM & 750 & 58.32 \\
\hline & \multicolumn{4}{|c|}{ XMM-Newton observatory. I. The spacecraft and operations } \\
\hline \multirow[t]{2}{*}{ 2010A\&A...517A..92A } & M. Arnaud et al. & EPN, EMOS & 157 & 46.72 \\
\hline & \multicolumn{4}{|c|}{$\begin{array}{l}\text { The universal galaxy cluster pressure profile from a representative } \\
\text { sample of nearby systems (REXCESS) and the YSZ - M500 relation }\end{array}$} \\
\hline \multirow[t]{3}{*}{ 2009A\&A...493..339W } & M. G. Watson et al. & EPN, EMOS & 202 & 41.56 \\
\hline & \multicolumn{4}{|c|}{ The XMM-Newton serendipitous survey. $V}$. \\
\hline & \multicolumn{4}{|c|}{ The Second XMM-Newton serendipitous source catalogue } \\
\hline \multirow[t]{2}{*}{$2005 \mathrm{~A} \& \mathrm{~A} \ldots 441 . .417 \mathrm{H}$} & G. Hasinger et al. & EPN, EMOS & 303 & 37.36 \\
\hline & \multicolumn{4}{|c|}{$\begin{array}{l}\text { Luminosity-dependent evolution of soft } X \text {-ray selected AGN. } \\
\text { New Chandra and XMM-Newton surveys }\end{array}$} \\
\hline \multirow[t]{3}{*}{ 2007АрJ...663...81P } & M. Polletta et al. & EPN, EMOS & 233 & 36.63 \\
\hline & \multirow{2}{*}{\multicolumn{4}{|c|}{$\begin{array}{l}\text { Spectral Energy Distributions of Hard X-Ray Selected Active } \\
\text { Galactic Nuclei in the XMM-Newton Medium Deep Survey }\end{array}$}} \\
\hline & & & & \\
\hline \multirow[t]{2}{*}{ 2001A\&A...365L...7D } & J. W. den Herder et al. & RGS & 421 & 32.74 \\
\hline & \multicolumn{4}{|c|}{ The Reflection Grating Spectrometer on board XMM-Newton } \\
\hline \multirow[t]{2}{*}{ 2009A\&A...498..361P } & G. W. Pratt et al. & EPN, EMOS & 143 & 31.59 \\
\hline & \multicolumn{4}{|c|}{$\begin{array}{l}\text { Galaxy cluster } X \text {-ray luminosity scaling relations } \\
\text { from a representative local sample (REXCESS) }\end{array}$} \\
\hline \multirow[t]{2}{*}{ 2003АрJ...590..207P } & J. R. Peterson et al. & RGS & 306 & 29.30 \\
\hline & \multicolumn{4}{|c|}{$\begin{array}{l}\text { High-Resolution X-Ray Spectroscopic Constraints } \\
\text { on Cooling-Flow Models for Clusters of Galaxies }\end{array}$} \\
\hline
\end{tabular}

of interest to a much larger scientific community.

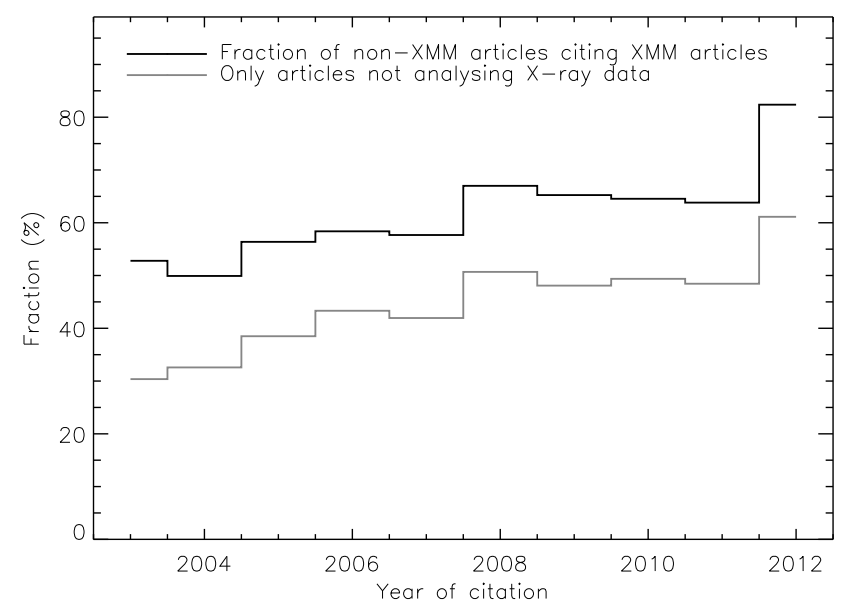

Fig. 4 The fraction of citing papers that do not directly use XMM-Newton data (black histogram). In addition, the same fraction is determined for papers that do not primarily discuss the analysis of X-ray data.

\subsection{Usage Statistics}

In order to put statistics on used observations into a context, the reference sample of useable observations was defined in Sect. 3, comprising 8577 pointed observations. We exclude slew exposures in the analysis that follows and instead performed a quick separate assessment: 2738 slew observations have been taken up to the end of 2012, 603 of these (22\%) have been used (excluding the article by Saxton et al. 2008), which is a high number considering that slew exposures are taken at random positions and at random times. 251 slew observations have been used more than once, up to five times, yielding a total of 926 slew observations that have been used in scientific articles including multiple use.

We now present results that rely both on bibliographic information from the ADS and on the instrument and ObsIDs determined by manual screening. The total number of papers and ObsIDs in the publication database are listed in Table 2. The distribution of the number of observations used in the papers is shown in Fig. 5. The distribution follows a power law with an index of -1.7 , yielding a median of 2.7 observations per paper. The majority of papers thus present the analysis of individual observations. In Fig. 5, we highlight sub-samples of $80 \%$ and $99 \%$ of papers with two shades of grey which analyse up to 9 and 290 observations, respectively. These two samples cover 


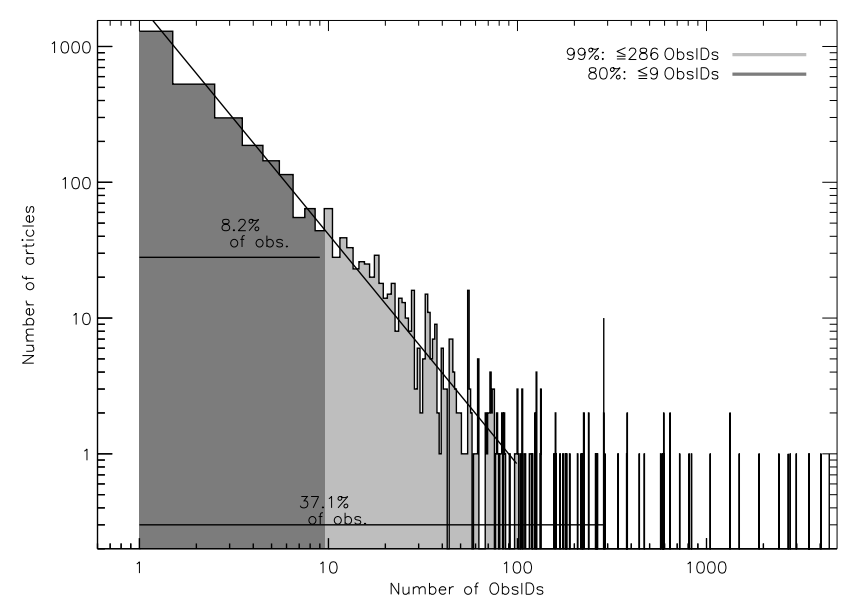

Fig. 5 The distribution of the number of observations used per paper follows an approximate power-law distribution with the majority of papers using data from less than three observations. $1 \%$ of XMM-Newton papers use data from more than 290 different observations. The shades mark accumulated fractions of all papers, as indicated in the legend. The vertical bars mark the limits given in the legend, and the percentages give the fraction of all observations used in the corresponding sub-samples of articles.

$8.1 \%$ and $37.2 \%$ of all observations in the database. $63 \%$ of observations have been used in $1 \%$ of articles making use of more than 290 ObsIDs each. In order to show potential differences between dedicated papers and those papers using large samples of data, some of the analysis presented in this section has been carried out separately for the full sample of articles and the 99-\% subsample of articles which use less than 290 observations.

\subsubsection{Time delay between observation and first publication}

In Fig. 6, we show the distribution of elapsed time between observation and the publication of the first article for GO and ToO observations. This study was performed for all papers excluding the 2XMM catalog by Watson et al. (2009) (open histograms) and excluding articles using more than 290 observations (99-\% subsample filled histograms). The sample sizes, i.e., the total number of observations under these two programme categories used in at least one paper, are given in the legends.

The distributions of $\mathrm{ToO}$ and $\mathrm{GO}$ programmes contain sharp peaks after approximately 1 and 2 years, respectively. ToO observations have a proprietary period of 6 months, and the faster publication of ToO observations reflects this. The presence of a second peak at 3.25 years in the distribution of publication times of GO observations is noteworthy. This occurs one year after the main peak and could reflect the one year proprietary period before public access to GO data. The presence of the second peak and the significant number of papers published many years after the data were taken indicates that archival research probably play an important role in the scientific success of XMM-Newton.

\subsubsection{Use of available science time}

In Fig. 7, we present the fraction of available science time for each observing cycle $(\mathrm{AO})^{4}$ that was used in at least one publication (top panel), in multiple publications (middle panel), and in multiple publications per AO (bottom panel) with percentages given on top of each bar. The 2XMM catalogue by Watson et al. (2009) is excluded. We also computed the same numbers excluding the $1 \%$ of papers which use more than 290 observations and show these results with the lighter grey bars. A lower fraction of observations taken in more recent AOs have been published which is unsurprising given the distribution of elapsed time between observation and first publication, illustrated in Fig. 6. More than $95 \%$ of observing time has been used in scientific publications, while round $80 \%$ of the time has been used in dedicated papers focusing on a few observations. The earliest observations have been recycled up to 20 times, where the fraction of multiple use scales with age of the data. The bottom panel shows the rate with which data were re-used per AO. Each kilosecond of observing time is used on average twice per year.

In Fig. 8, we illustrate the distribution of multiple usage based on the full publication database with the open histogram. The observation with the highest recycling rate is ObsID 0097820101 (the Galaxy Cluster A 1795) which has been used in 67 articles. The exclusion of papers using large numbers of observations in one project naturally reduces the recycling rate and is shown in Fig. 8 with the grey histogram. The observation with the highest recycling rate in the reduced sample is also ObsID 0097820101 being used 51 times. Also without these papers, an impressively large number of observations has been used multiple times.

\subsubsection{Science categories}

The majority of XMM-Newton observations were taken in the Guest Observer (GO) programme. The GO programme is composed of regular observations of certain targets with observing times up to $300 \mathrm{ksec}$, Large Programs $(>300 \mathrm{ksec})$, and triggered observations taken in response to anticipated events. In addition, Target of Opportunity (ToO) observations are performed in response to unanticipated events. Finally, calibration observations are included at regular intervals to monitor the performance of each instrument with data immediately available to the scientific

\footnotetext{
${ }^{4}$ Announcement of Opportunity cycles (AOs) last one year and start on May 1st of each year
} 


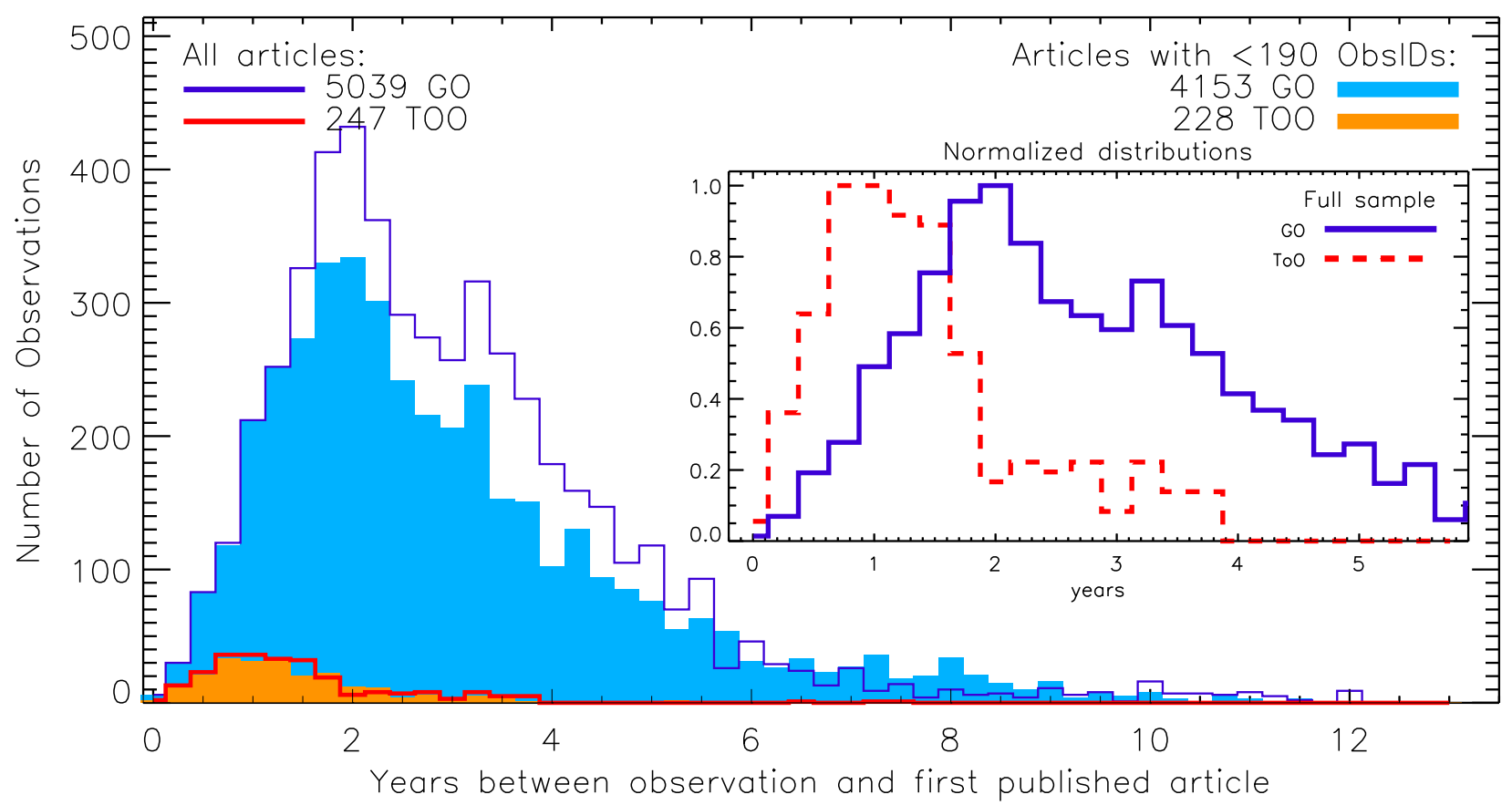

Fig. 6 Distribution of elapsed time between observation and publication dates of the first article using them. The analysis was performed for programme categories Guest Observer (GO) and Targets of Opportunity ( $\mathrm{ToO}$ ), shown with different colours. The 2XMM catalogue (Watson et al. 2009) is excluded from this analysis. The shaded distributions represent the results from the reduced database, excluding $1 \%$ of articles that use more than 290 ObsIDs. The inset shows the normalised distribution, illustrating that ToOs are published faster than regular GO observations.

community.

In Fig. 9, we show the time evolution of the fraction of available observations that have been used in at least one article for GO observations (open histogram). Observations that have been used in the Watson et al. (2009) are excluded to avoid overestimation of the usage. The light shaded area indicates the same results for GO observations having only been used in articles analysing less than 290 observations. The last three years yield a lower usage presumably because many observations are still being analysed (see Fig. 6).

Excluding observations taken within the last three years, $92 \%$ of GO observations have been used to produce a refereed XMM-Newton paper. About $76 \%$ of all observations have been used in papers which analyse less than $290 \mathrm{ob}-$ servations. In the bottom left legend of Fig. 9, we give the same average numbers for the other programmes. $82 \%$ of calibration observations have been used in all papers combined (excluding only Watson et al. 2009) and 65\% in papers with less than 290 observations, respectively.

\subsubsection{Instrument usage}

We studied the instrument usage of all papers except those describing the instruments themselves. $78 \%$ of them focus on data from a single instrument, while $19 \%$ use data from two instruments, and $3.4 \%$ use data from all three instruments. The majority (96\%) of papers use data from one or both EPIC detectors. The RGS spectrometer can only be used for bright point-like sources, naturally leading to less usage (18\%). Data from the optical monitor (OM) was used in $11 \%$ of all articles.

The importance of the results obtained with each instrument can be measured in terms of the citation rates of the articles using their data, computed in the same way as in Sect.4.1.4.

The evolution of the citation rate for papers analysing data from different instruments is illustrated in Fig. 10. The total sample sizes are given in the right legend and comprise papers using the given instruments alone or in combination with data from other instruments.

All instruments contribute roughly equally to the high overall citation rate discussed in Sect. 4.1.4 The citation rate of the earliest RGS articles is notably higher. Although the brightest sources had quickly been observed with the RGS, later articles analysing RGS data still receive high citation rates above three citations per article per year. Also those articles making use of the OM receive comparably high citation rates.

\subsubsection{Science categories}

Each observation carried out under the GO and ToO programmes is assigned a science category by the investigator, 


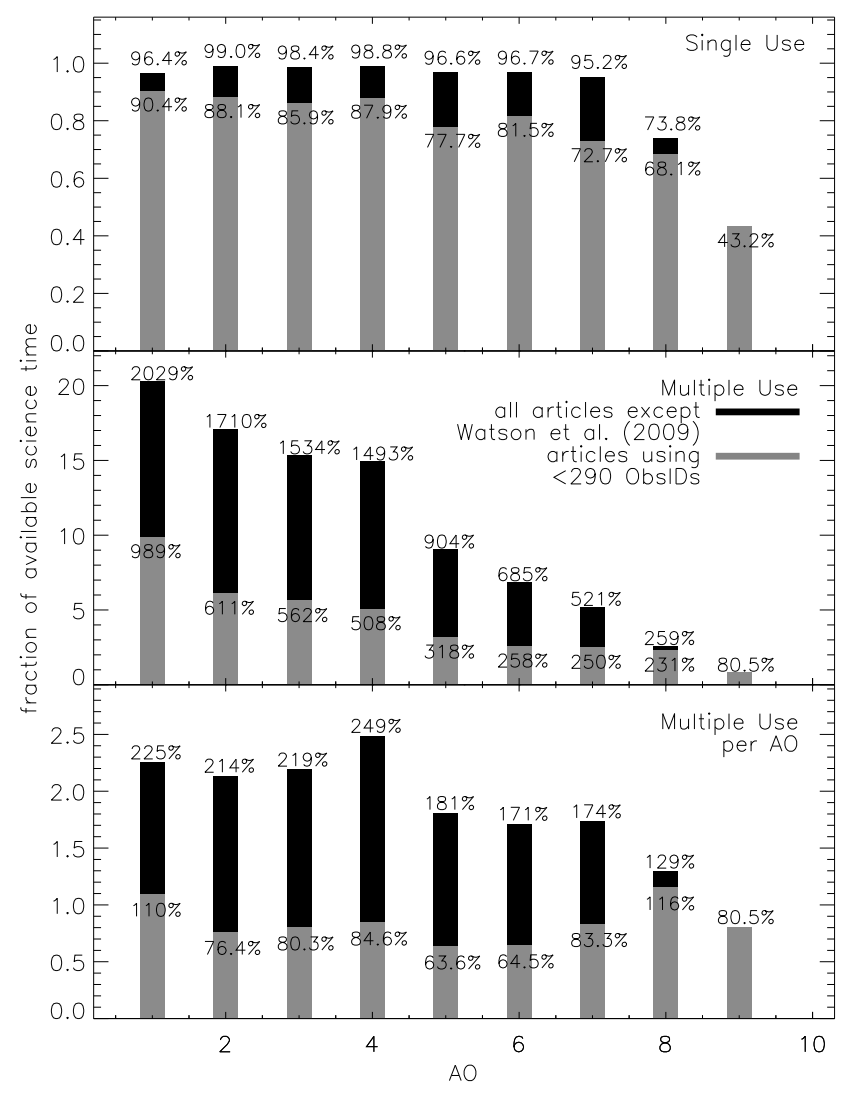

Fig. 7 The fraction of available science time used in at least one publication (top), accounting for multiple use (middle), and multiple use per observing cycle (AO; bottom). Multiple use is accounted for by multiplication of the observing time from each observation by the number of papers using it. For the black bars, the 2XMM catalogue Watson et al. 2009) is excluded while for the grey bars, all papers using more than 290 observations are excluded. Multiple use naturally increases with the age of the data (middle panel), yielding a roughly constant recycling rate (bottom).

following the HEASARC Object Classifications scheme 5 . For calibration observations, the assignments are given by the instrument team selecting the targets. We combined these codes into 21 rough groups to study the use of data taken under different science categories. The grouping is shown in Table 5 where the names of science categories are listed in the left column, the ranges of HEASARC class codes in the second column, and the number of available observations in the XSA in the last column. We defined an additional category (19) for Cosmology and Deep Field projects, including all observations with target names Marano, Lockman, CDF, AXAF, HDF, COSMOS, MLS, SZE, Groth-Westphal, or Deep Field.

For each science category listed in Table 5, the fraction of observations that have been used in scientific articles is shown in the top panel of Fig. 11, with percentages given

5 http://heasarc.gsfc.nasa.gov/W3Browse/class_help.html

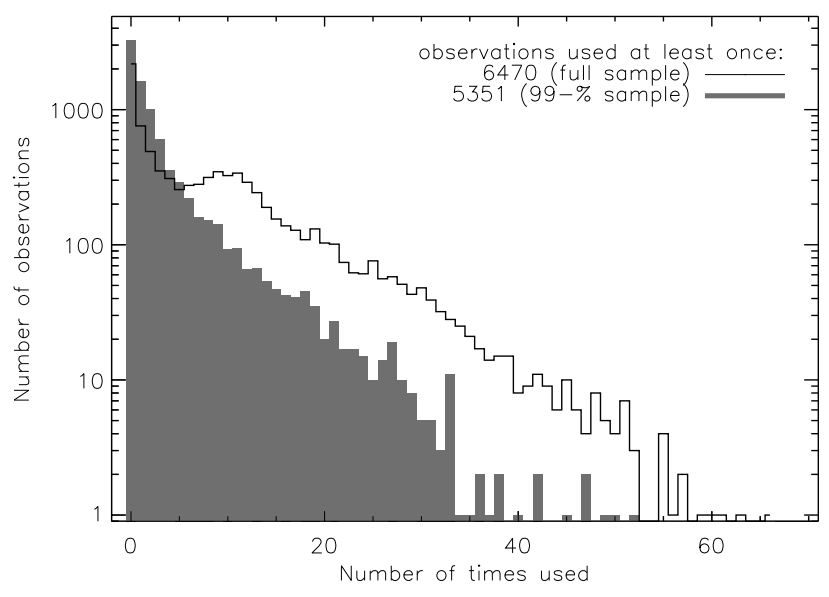

Fig. 8 Multiple use of observations used at least once. The horizontal axis shows the number of papers in which the same observation was used while the vertical axis counts the number of observations that has been used in as many papers given by the horizontal axis. The grey histogram shows the same distribution for the $99 \%$ subsample of articles using less than 290 observations.

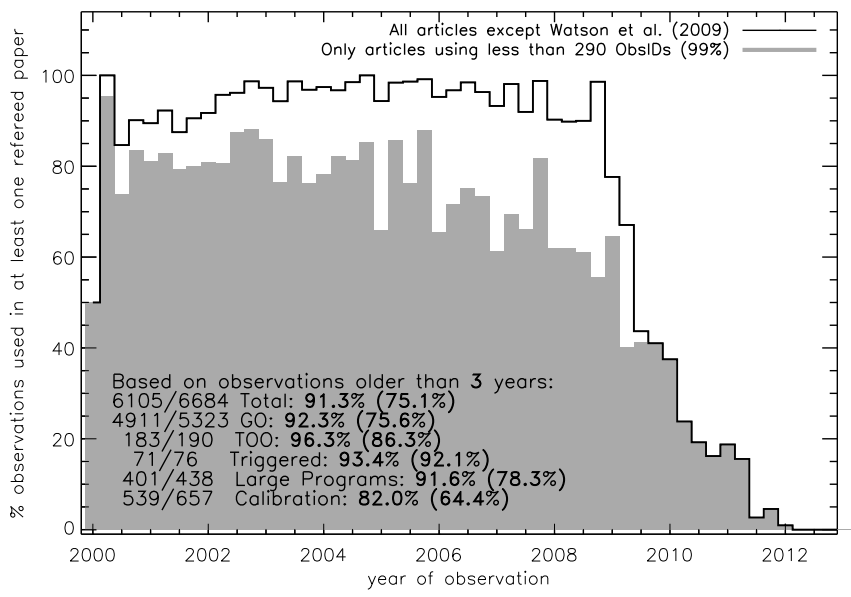

Fig.9 The percentages of observations that have been used in scientific papers as of end-2012. The black open histogram accounts for all papers, excluding only observations used by Watson et al. (2009) whilst the light shaded histograms also exclude observations used in $1 \%$ of papers that have analysed more than 290 ObsIDs. The plot shows the results for regular GO observations (see text). Average usage for other program categories is given in the lower left corner, where only observations older than three years are included in the average. Given are, for each program, number of observations used versus number of usable observations, percentage of total sample and, in brackets, the same fraction resulting from accounting only for paper from the partial sample of papers using less than 290 ObsIDs.

on top. The numbers in the bottom refer to those in the first column of Table 5. The recycling rate is shown in the bottom panel, where multiple use is taken into account. The histograms show that each observation was used on average 


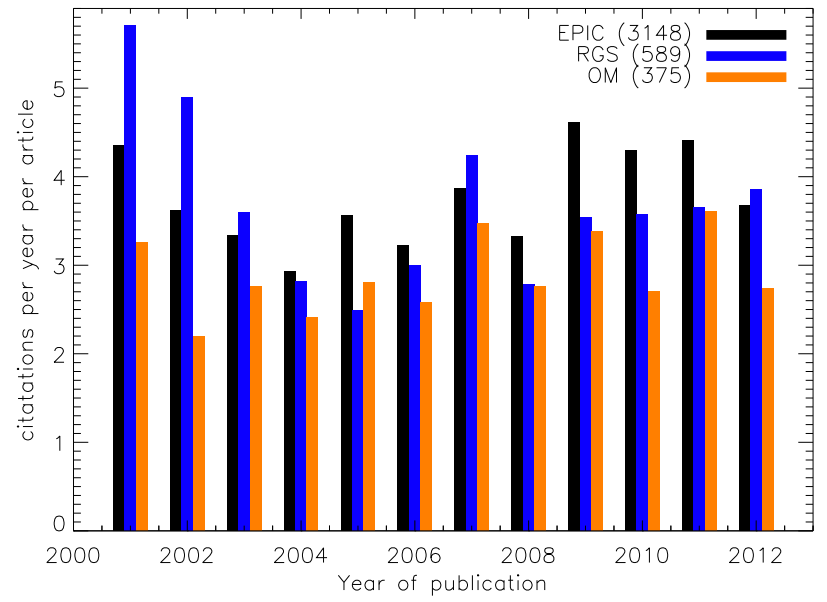

Fig. 10 Citation statistics of articles using EPIC, RGS, and OM data (excluding instrument papers; see legend). The sample includes 3274 articles, excluding papers describing the instruments. Similarly high impact was achieved by all three instruments.

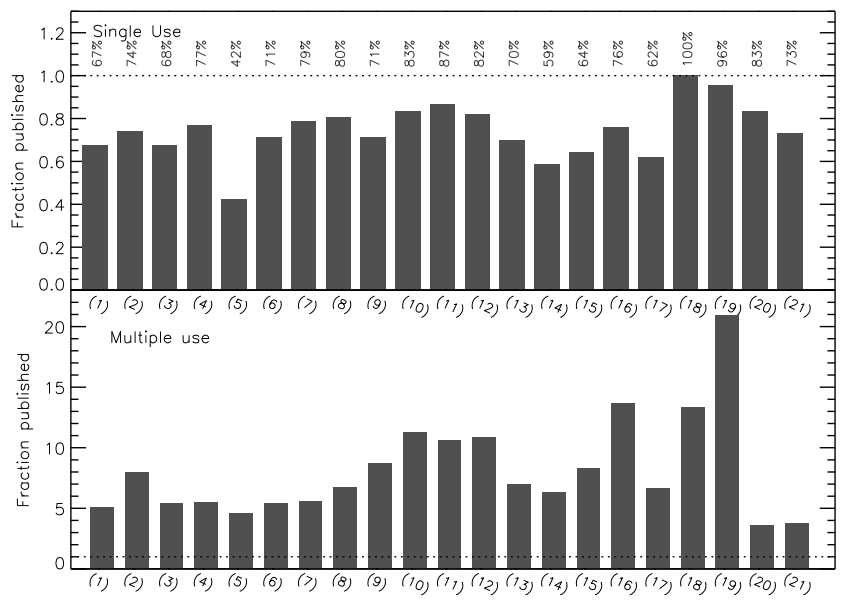

Fig. 11 Fractions of observations used in at least one refereed paper (top panel) for each of the science categories listed in Table 5. In the bottom panel, observations used more than once are counted accordingly multiple times. The fractions in top and bottom are relative to the total numbers of observations in each category, given in the last column of Table 5

more than five times. The highest recycling rate is in the Cosmology/Deep field category.

\section{Summary and conclusions}

XMM-Newton, ESA's second X-ray observatory, was launched in 1999 and since then has been providing the scientific community with high quality imaging and spectroscopy. The annual calls for observing proposals are typically over-subscribed by a factor 6 in observing time. In order to investigate the scientific productivity of XMMNewton, we have created a database currently containing information about refereed papers that directly use XMM-
Table 5 Science categories used in Fig. 11

\begin{tabular}{|c|c|c|}
\hline Science Category & Class codes $^{a}$ & $\# \mathrm{obs}^{6}$ \\
\hline (1) Solar System object & $8000-8999$ & 46 \\
\hline (2) Extrasolar Planet/Brown dwarf/Protostar & $1850-1899$ & 65 \\
\hline (3) Stars/WDs & & 1062 \\
\hline- Stars & $1900-2999$ & 960 \\
\hline - White dwarfs & $4000-4999$ & 102 \\
\hline (4) Binaries & & 1032 \\
\hline - Cataclysmic Variables & $1600-1699$ & 341 \\
\hline - X-ray binary/QPO & $1000-1599$ & 691 \\
\hline (5) Black Holes & & 78 \\
\hline- ULX & $9300-9399$ & 61 \\
\hline - Supermassive black hole & $9400-9499$ & 17 \\
\hline (6) Gamma ray source & $1700-1799$ & 161 \\
\hline (7) Pulsar/Neutron star & $1800-1849$ & 478 \\
\hline (8) SNR/Nebulae/Diffuse emission & 3000-3999 & 1005 \\
\hline (9) Galaxies & $6000-6999$ & 760 \\
\hline (10) Active Galaxies & & 1107 \\
\hline - AGN, Seyferts & $7000-7199$ & 869 \\
\hline - AGN (Liners) & $7400-7499$ & 26 \\
\hline - Radio/IR Galaxies & 7600-7999 & 212 \\
\hline (11) QSO & $7200-7299$ & 452 \\
\hline (12) BL Lac & 7300-7399 & 182 \\
\hline (13) Radio galaxies & 7500-7599 & 182 \\
\hline (14) Groups of Galaxies & $5200-5299$ & 182 \\
\hline (15) Clusters of Galaxies & $5000-5099$ & 1152 \\
\hline (16) X-ray background & $5500-5599$ & 117 \\
\hline (17) Supernovae and Hypernovae & 9100-9299 & 21 \\
\hline (18) Unusual Object & 9000 & 3 \\
\hline (19) Cosmology / deep fields & arget Names ${ }^{c}$ & 209 \\
\hline (20) Unidentified & 9999 & 77 \\
\hline \multirow[t]{2}{*}{ (21) Unclassified } & & 111 \\
\hline & Total: & 8482 \\
\hline
\end{tabular}

${ }^{a}$ http://heasarc.gsfc.nasa.gov/W3Browse/class_help.html

${ }^{b}$ Number of observations containing at least one science exposure ${ }^{c}$ All observations with target names containing Marano, Lockman, CDF, AXAF, HDF, COSMOS, MLS, SZE, Groth-Westphal, or Deep Field

Newton data and the observations that were used. We analysed 3272 articles published until end of 2012 to provide the results reported in these papers. This information was obtained from the SAO/NASA Astrophysics Data System (ADS) and the XMM-Newton Science Archive (XSA). The main conclusions of this study are:

- Following launch in 1999, the annual number of XMMNewton refereed papers rose to 300 per year by 2003 or 2004 and has remained approximately constant with no evidence for a decrease in recent years. This continued high level of scientific productivity is remarkable for a mission that is in its 13th year of operation with essentially the same scientific performance as at launch. It is worth noting that the continued high oversubscription factor in requested observing time supports the view that XMM-Newton continues to remain competitive with other facilities. 
- Approximately 100 scientists publish for the first time as first authors of refereed XMM-Newton papers each year and about 500 appear as co-authors. The large and unchanging number of new scientists working with XMMNewton, suggests that the X-ray community continues to attract new members who are able to successfully analyse and publish results from the mission.

- On average, each XMM-Newton paper receives four citations each year. The long-term citation rate, counting citations given more than a few years after publication, remains at a high average level of three citations per year for each article, five years after publication. About two thirds of all citations originate from articles that are not in the XMM-Newton database, thus originate from research that is not primarily the analysis of XMM-Newton data. Some $50 \%$ of citing articles do not mention other major X-ray missions, and both citation rates demonstrate the broad relevance of XMM-Newton results, beyond the $\mathrm{X}$-ray community itself.

- The impact of data from all three instruments is roughly the same in terms of average citation rate.

- More than 90\% of XMM-Newton observations taken so far have been used in a scientific publication. Some $10 \%$ of observations have only been used in projects using several hundred of observations which are probably surveys. Even calibration observations, that were not primarily taken for scientific purposes, and random slew observations have been used at levels of $82 \%$ and $22 \%$, respectively. An impressively large number of observations has been used multiple times, indicating the importance of XMM-Newton data beyond the initial motivation.

- The median time between GO and the first refereed paper being published ranges from a few months up to several years with the maximum around two years. We found evidence for a second peak in the distribution of elapsed times at 3.25 years, almost exactly one year after the first peak may reflect the one year proprietary period. ToO observations are published significantly faster with a median time of one year.

Acknowledgements. This project benefitted from resources kindly provided by the XMM-Newton community support and scientific planning team. We thank Stefan Immler (NASA), Nuria Fonseca Bonilla, Sara Bertran de Lis, and Pablo Ramirez Moreta for support during screening of articles. The project was kindly supported by Steve Snowden (NASA Project scientist). We thank Lauren Boice for useful discussions. This research has made use of NASA's Astrophysics Data System Bibliographic Services.

\section{References}

Apai, D., Lagerstrom, J., Reid, I. N., et al. 2010, PASP, 122, 808

den Herder, J. W., Brinkman, A. C., Kahn, S. M., et al. 2001, A\&A, 365, L7

Heck, A. 2006, Organizations and Strategies in Astronomy, Vol. 7, 335

Jansen, F., Lumb, D., Altieri, B., et al. 2001, A\&A, 365, L1
Mason, K. O., Breeveld, A., Much, R., et al. 2001, A\&A, 365, L36

Rots, A. H., Winkelman, S. L., \& Becker, G. E. 2012, PASP, 124, 391

Saxton, R. D., Read, A. M., Esquej, P., et al. 2008, A\&A, 480, 611

Strüder, L., Briel, U., Dennerl, K., et al. 2001, A\&A, 365, L18

Talavera, A. 2009, APSS, 320, 177

Trimble, V. 1986, PASP, 98, 1347

Turner, M. J. L., Abbey, A., Arnaud, M., et al. 2001, A\&A, 365, L27

Watson, M. G., Schröder, A. C., Fyfe, D., et al. 2009, A\&A, 493, 339 